\title{
Estradiol postconditioning relieves ischemia/reperfusion injury in axial skin flaps of rats, inhibits apoptosis and alters the MKP-1/ERK pathway
}

\author{
QIANHENG JIN ${ }^{1}$, JIHUI JU ${ }^{1}$, LEI XU ${ }^{1}$, YUEFEI LIU ${ }^{1}$, ZHIMIN LI ${ }^{1}$, YI FU ${ }^{2}$ and RUIXING HOU ${ }^{1}$ \\ ${ }^{1}$ Institute of Hand Surgery, Ruihua Affiliated Hospital of Soochow University; \\ ${ }^{2}$ Department of Human Anatomy, Histology and Embryology, School of Biology and \\ Basic Medical Sciences, Soochow University, Suzhou, Jiangsu 215100, P.R. China
}

Received March 21, 2016; Accepted March 10, 2017

DOI: $10.3892 / \mathrm{mmr} .2017 .6708$

\begin{abstract}
Previous studies have suggested that estradiol can reduce the ischemia/reperfusion (I/R) injury in skin flaps. However, the mechanism, particularly the signal pathways involved in this protective effect are not well established. In the current study, an I/R injury model was established in rats to explore the connection between estradiol protection during I/R injury and extracellular signal-regulated kinase (ERK) signaling. Healthy male Wistar rats were divided into five groups (n=10): Control group (group I), I/R group (group II), saline group (group III), estradiol group (group IV) and inhibitor (PD-98059) group (group V). The survival rate of the flap was compared between groups, morphological changes were observed by hematoxylin and eosin staining of sections, and terminal deoxynucleotidyl transferase dUTP nick end labeling was performed to identify apoptotic cells and determine the apoptotic index. To further investigate the mechanism, western blot analysis was performed to assess the protein level of $\mathrm{ERK}_{1 / 2}$, phospho-ERK $\mathrm{E}_{1 / 2}$, and mitogen-activated protein kinase phosphatase 1 (MKP-1). The results of the present study demonstrated that estradiol therapy can reduce I/R injury and decrease the apoptosis index in an axial skin flap model. The inhibitor of the ERK pathway (PD-98059) partially abolished the effects of estradiol, which involve the phosphatase enzyme MKP-1. Taken together, the findings of the present study
\end{abstract}

Correspondence to: Professor Ruixing Hou, Institute of Hand Surgery, Ruihua Affiliated Hospital of Soochow University, 5 Tayun Road, Suzhou, Jiangsu 215100, P.R. China

E-mail: hourx@rxhospital.com

Professor Yi Fu, Department of Human Anatomy, Histology and Embryology, School of Biology and Basic Medical Sciences, Soochow University, 199 Renai Road, Suzhou, Jiangsu 215100, P.R. China

E-mail: yfu@suda.edu.cn

Key words: estradiol, axial flaps, ischemia-reperfusion injury, extracellular signal-related kinase pathway, apoptosis indicate that estradiol may act by reducing the expression of MKP-1, mediating the expression/activation changes of the ERK pathway and subsequently reduce the level of apoptosis and the I/R injury the axial flap. Estrogen may be used to mitigate the adverse reaction caused by ischemia-reperfusion injury and effectively improve the survival rate and survival quality of free skin flap and improve patient prognosis.

\section{Introduction}

Skin flap transplantation is one of the most commonly used, and effective methods to repair tissue defects and reconstruct organs. However, the treatment is impeded due to the occurrence of ischemia/reperfusion (I/R) injury in the process of transplantation, which produces toxic compounds, including lipid peroxide and reactive oxides. Thus, patients may require longer hospitalization, multiple unexpected surgeries and increased costs (1). A number of studies have reported that I/R injury of skin flaps caused a series of complex pathophysiological processes mediated by various cell types and factors. The main mechanisms may involve the production of reactive oxygen species (ROS), the adhesion and aggregation of neutrophils, calcium overload, apoptosis and other processes (2-6). Apoptosis is considered to be an important factor in $\mathrm{I} / \mathrm{R}$ injury of skin flaps (7). The mechanism of I/R damage may involve the excessive production of oxygen free radicals and the cross-links between proteins, resulting in dysfunction of proteins with enzyme activity and altered gene transcription $(8,9)$.

Extracellular signal-regulated kinase (ERK) is an important downstream mediator in the mitogen-activated kinase (MAPK) pathway, with a crucial role in cellular signal transduction pathways involved in cell proliferation, differentiation, morphology, cytoskeleton construction, apoptosis, and carcinogenesis $(10,11)$. Previous research had demonstrated that the ERK signaling pathway is closely associated with I/R injury (12). Darling et al (13) reported that the protective effect of myocardial ischemia in the heart tissue of rabbits was mediated by the ERK pathway, rather than the phosphoinositide 3-kinase pathway. Jung et al (14) reported that troglitazone induces apoptosis of various types of cells, 
which may be linked to inhibition of the ERK pathway. Therefore, I/R injury in skin flaps may be mediated by the ERK pathway. Mitogen-activated protein kinase phosphatase 1 (MKP-1), which is also termed dual specificity phosphatase 1 , is a specific negative regulator of the ERK pathway, and can block substrate activity by promoting the serine/threonine or tyrosine dephosphorylation (15).

The current treatment measures for I/R injury of skin flaps include pretreatment of the skin flap, drug treatment (growth factor, L-selection, calcium antagonists), biological technology (antibodies, gene therapy) and sound wave therapy (16). Drug treatments have the best prospects for clinical application. The hormone estrogen has received attention for its potential use in the therapy of I/R injury. Currently, research findings have demonstrated that minute quantities of estrogen can alleviate the I/R injury of skin flaps; the effect may be mediated by NO production in vascular endothelial cells, anti-oxygenation and anti-apoptosis (17). The underlying mechanism of estradiol postconditioning protection remains incompletely understood. In order to determine whether the application of estradiol would be useful for the clinical treatment of I/R injury, the current study was designed investigate the association between estradiol, apoptosis and the ERK signaling pathway.

\section{Materials and methods}

Ethics. All experimental procedures and protocols used in this investigation were reviewed and approved by the Animal Care and Use Committee of the Experimental Animal Center of Soochow University (Suzhou, China), and conformed to the Guide for the Care and Use of Laboratory Animals published by the US National Institutes of Health. The researchers that performed the animal experiments, possessed qualified certification issued by the Department of Science and Technology of Jiangsu Province.

Materials. Adult, male Wistar rats (250-350 g) were obtained from the specific pathogen-free laboratory of the Experimental Animal Center of Soochow University, were housed with $12 / 12 \mathrm{~h} \mathrm{light/dark} \mathrm{cycle} \mathrm{in} \mathrm{a} \mathrm{temperature-controlled} \mathrm{room} \mathrm{and}$ had free access to food and water. Estradiol was purchased from Sigma-Aldrich (Merck KGaA, Darmstadt, Germany). $\mathrm{ERK}_{1 / 2}$ rabbit monoclonal antibody mAb (cat. no. 4348S), phospho-ERK ${ }_{1 / 2}$ rabbit $\mathrm{mAb}$ (cat. no. 4370S) and PD-98059 (cat. no. 9900L) were all obtained from Cell Signaling Technology, Inc. (Danvers, MA, USA). MKP-1 antibody (cat. no. ab1351) was obtained from Abcam (Cambridge, UK). Secondary antibodies were goat anti-rabbit (cat. no. A0239), donkey anti-goat (cat. no. A0266), goat anti-rat (cat. no. A0192) and GAPDH (cat. no. AG019) were all obtained from Beyotime Institute of Biotechnology (Shanghai, China). A terminal deoxynucleotidyl transferase dUTP nick end labeling (TUNEL; cat. no. 11684795910) staining kit, which was used to detect apoptotic index, was purchased from Roche Diagnostics (Basel, Switzerland).

Animal model establishment. Wistar rats were anesthetized by intraperitoneal injection of $2 \%$ pentobarbital sodium $(20 \mathrm{ml} / \mathrm{kg})$. The rats were put into a supine position with the neck extended and the area was shaved in preparation for the procedure. The operation area was sterilized with iodophor and covered with sterile surgical towel. As previously described by Manson et al (18), an axial pattern skin flap of 3x6 cm in size was made of each rat, with the superficial artery and vein in the abdominal wall acting as the pedicle. The blood flow of the flap was blocked completely by a microvascular clip to clamp the vessel pedicle for $6 \mathrm{~h}$ and the flap was sutured to their original position with 5-0 stitches. During the period of the ischemia, anesthesia with was maintained (2\% pentobarbital sodium). After $6 \mathrm{~h}$ of ischemia, the clamp was removed and the $\mathrm{I} / \mathrm{R}$ injury model has been established.

Experimental groups. Rats were fed to $>250 \mathrm{~g}$ and divided randomly into five groups ( $n=10$ per group) as follows: Group I (control group), after the flap was harvested, it was sutured to its own donor site without clamps to reduce blood flow; Group II (I/R group), the I/R injury model was established with no treatment; Group III (saline group), the I/R injury model was established and normal saline $(2 \mathrm{ml} / \mathrm{kg}$ ) injected; Group IV (estradiol group), the I/R injury model was established treated with estradiol (100 $\mu \mathrm{g} / \mathrm{kg}$ ) injected; Group V (inhibitor group), $\mathrm{I} / \mathrm{R}$ model rats were co-treated with estradiol $(100 \mu \mathrm{g} / \mathrm{kg})$ and PD-98059 which may inhibit Mek-1, the upstream protein of Erk $(0.3 \mathrm{mg} / \mathrm{kg})$. Rats of groups III-V were treated with saline, estradiol, PD-98059 and estradiol by intraperitoneal injection in the right lower abdomen after the surgery, and at day 2, 4 and 6 postoperatively.

Observation of the skin flap. The gross condition of the skin flap was observed at days 2, 4 and 6 postoperatively. The color, hair growth, survival area and the inflammation exudation of the flaps were monitored by daily observation.

Determination of flap survival. Images of the flaps were captured using a digital camera at 7 days postoperatively, and computerized planimetry was performed using Image-Pro Plus v6.0 (Media Cybernetics, Inc., Rockville, MD, USA). The necrotic area was recorder, which was obviously different in color compared with the normal tissue. The survival rate of the flap was obtained by dividing the survival area of the skin flap by the total area of the flap.

Histology analysis. The rats were anesthetized at 7 days postoperatively. A full-thickness skin sample of $0.5 \times 0.5 \mathrm{~cm}$ in size near the vascular pedicle was obtained and fixed in $10 \%$ formalin overnight. The sections were dehydrated in alcohol, rehydrated in xylene, and embedded in wax. $10 \mu \mathrm{m}$ consecutive section, label for each slice, and standby, a normal slice was manufactured. The sections were stained with hematoxylin and eosin. The histological characteristics of the tissues were evaluated under a light microscope, 10 fields of view were captured per slice.

Apoptosis index. Apoptosis was determined by TUNEL assay in each rat. The skin flap tissues were sectioned into $10 \mu \mathrm{m}$ by using a microtome. Sections were dewaxed in xylene for $10 \mathrm{~min}$ and dehydrated in an ascending ethanol gradient. After preparation of washing, the slices were soaked in the TUNEL reaction solution at room temperature for $1 \mathrm{~h}$. Subsequently, the slices were washed in PBS for 3 times for $5 \mathrm{~min}$, fixed in $4 \%$ 
paraformaldehyde for $15 \mathrm{~min}$ and sealed by methanol containing $3 \% \mathrm{H}_{2} \mathrm{O}_{2}$ for $10 \mathrm{~min}$ at room temperature. Finally, after being immersed in the diaminobenzidine for $5 \mathrm{~min}$, the sections were stained with hematoxylin and observed under a light microscope. For each TUNEL-stained section, three randomly selected high power fields were imaged. Apoptotic nuclei were deeply brown stained. Three random high-power fields were imaged from each section. Apoptotic and non-apoptotic cells were identified by Image Pro Plus version 6.0 software (Media Cybernetics, Inc., Rockville, MD, USA). The apoptotic index was equal to apoptotic cells divided by the total cells.

Western blot analysis. At 7 days after the surgery, an area of the skin flap near the vascular pedicle was harvested. The skin layer and adipose tissue were removed, and the tissue was cut into pieces and stored in a cryogenic refrigerator $\left(-80^{\circ} \mathrm{C}\right)$. RIPA lysis buffer and protease inhibitors were added to the tissues (100:1), and the samples were incubated on ice, centrifuged at $12,000 \mathrm{x}$ g for $10 \mathrm{~min}$ at $4^{\circ} \mathrm{C}$ and the supernatant extracted $30 \mathrm{~min}$ after centrifugation. Subsequently, the protein lysates (50 mg/lane) were loaded into 12\% SDS-PAGE gels (Beyotime Institute of Biotechnology), separated by electrophoresis and transferred to a nitrocellulose membrane. The membrane was blocked in 5\% non-fat powdered milk at room temperature with gentle shaking for $2 \mathrm{~h}$ and then washed in TBS-Tween 20 three times for $10 \mathrm{~min}$ each. Subsequently, the membrane was incubated with diluted primary antibody Erk $(1: 1,000$; cat. no. 4348S; Cell Signaling Technology, Inc.), p-Erk (1:1,000; cat. no. 4370S; Cell Signaling Technology, Inc.), MKP-1 (1:1,000; cat. no. ab1351; Abcam), GAPDH (1:1,000; cat. no. AG019; Beyotime Institute of Biotechnology) in 5\% $\mathrm{BSA}$ at $4^{\circ} \mathrm{C}$ overnight with gentle shaking. The membrane was washed by TBS-Tween for three times for $10 \mathrm{~min}$ each, then incubated with secondary antibody (Erk, p-Erk with goat anti-rabbit; 1:1,000; cat. no. A0239; Beyotime Institute of Biotechnology; MKP-1 with donkey anti-goat; 1:1,000; cat. no. A0266; Beyotime Institute of Biotechnology; GAPDH with goat anti-rat; 1:1,000; cat. no. A0192; Beyotime Institute of Biotechnology) for $1.5 \mathrm{~h}$ at room temperature. Finally, the proteins were detected by the method of ECL color (BeyoECL Plus, cat. no. P0018; Beyotime Institute of Biotechnology) and all the target protein bands were analyzed using Image $\mathrm{J}$ software (National Institutes of Health, Bethesda, MD, USA).

Statistical analysis. All the statistics were analyzed with SPSS software 17.0 (SPSS, Inc., Chicago, IL, USA). Experimental results are presented as the mean \pm standard deviation. One-way analysis of variance was used to determine the statistical difference among the groups, and Levene test was used for the evaluation of homogeneity of variances. The Tukey-Kramer test was used for pairwise comparison and the Bonferroni test was used for multiple comparisons. Pearson coefficient was used to analysis the flap survival rate and apoptosis index correlation. $\mathrm{P}<0.05$ was considered to indicate a statistically significant difference.

\section{Results}

Observation of the skin flap. Following the surgery to establish $\mathrm{I} / \mathrm{R}$, different degrees of swelling were observed in the I/R skin flap model rats and the skin appeared a violet color. Necrosis gradually emerged in the skin flaps. Necrotic areas appeared that were black, hard and had no hair growth in the I/R group and saline group. Compared to the original skin, the thickness of skin flap of I/R group and saline group increased significantly. However, the degree of swelling gradually declined in the control group, estradiol group and inhibitor group. Only a small are of necrosis appeared in the suture edge and the skin flap had no obvious differences to the normal skin in these three groups.

Flap survival rate determination. On postoperative day 7 , the skin flaps were images (Fig. 1A). Quantification of the skin flap survival rate is presented in Fig. 1B. The survival rates of the flap were $98.13 \pm 2.67 \%$ in the control group, $67.36 \pm 22.94 \%$ in the I/R group, $68.19 \pm 21.11 \%$ in saline group, $94.05 \pm 9.27 \%$ in the estradiol group and $78.87 \pm 15.65 \%$ in the inhibitor group. Compared with the control group, the survival rate of the flap was significantly decreased in the $I / R$ group $(\mathrm{P}<0.05)$. There was no significant change in the survival rate in the I/R skin flap model rats that received saline (saline group) and those that were untreated ( $\mathrm{I} / \mathrm{R}$ group; $\mathrm{P}>0.05$ ). The survival rate of the estradiol group was significantly higher than the I/R group $(\mathrm{P}<0.05)$. Additionally, the survival rate was significantly higher in the estradiol group than in the inhibitor group $(\mathrm{P}<0.05)$.

Histological analysis. At 7 day the I/R model was established, the tissue structure of the control group was normal, with cells arranged orderly and almost no observable infiltration of inflammatory cells. The following features were observed in the I/R and saline groups: Stratum corneum exfoliation, tissue edema, massive neutrophil aggregation, adhesion, loss of normal tissue structure and skin tissue necrosis. In the estradiol and inhibitor groups, the flap tissue inflammation and edema were mild, and the tissue structure was normal (Fig. 2).

Apoptosis index. TUNEL staining was used to detect apoptosis and the TUNEL-positive cells were stained brown. The apoptotic index was $5.69 \pm 1.475 \%$ in the control group, $45.02 \pm 8.128 \%$ in the I/R group, $49.18 \pm 5.801 \%$ in the saline group, $11.89 \pm 2.721 \%$ in the estradiol group and $18.95 \pm 4.04 \%$ in the inhibitor group. Compared with the control group, the apoptosis index was significantly increased in flap sections from the I/R group $(\mathrm{P}<0.05)$. Compared with the index in the I/R group, apoptosis was obviously decreased by estradiol treatment, and furthermore, the ERK inhibitor PD-98059 partially eliminated the effect of estradiol (Fig. 3).

Western blot analysis. The levels of ERK pathway proteins, ERK, p-ERK and MKP-1, were detected by western blotting. Compared with the I/R group, the levels of ERK were increased in the estradiol group, while the level of MKP-1 was decreased. PD98059 (inhibitor group) inhibited the increased activation of ERK (phosphorylation), but did not affect the protein expression of ERK and MKP-1. Unexpectedly, the levels of ERK and MKP-1 in the I/R group and saline group were marginally increased compared with the control group, which may be cause by the stress reaction to $I / R$ injury (Fig. 4). 
A
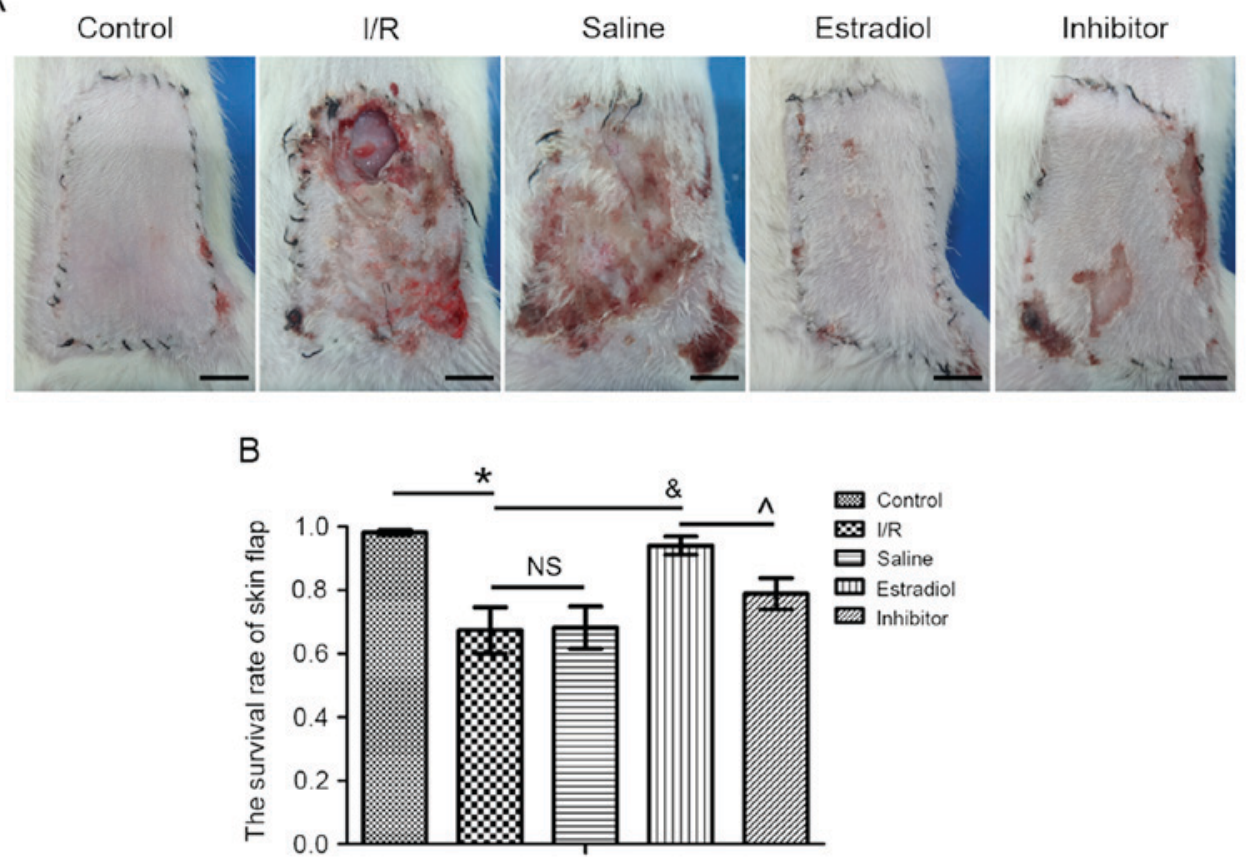

Figure 1. (A) Images of skin flaps captured on postoperative day 7, tissue and necrosis of skin flap were observed (scale bar, $1 \mathrm{~cm}$ ). (B) The skin flap survival rate of all groups. Necrotic area and survival area were identified by Image Pro Plus 6.0 software. Values are expressed as the mean \pm standard deviation. $\mathrm{n}=10$ per group; " $\mathrm{P}<0.05$ vs. control; ${ }^{\&} \mathrm{P}<0.05$ vs. I/R; ${ }^{\wedge} \mathrm{P}<0.05$ vs. estradiol. I/R, ischemia/reperfusion; NS, no significance.

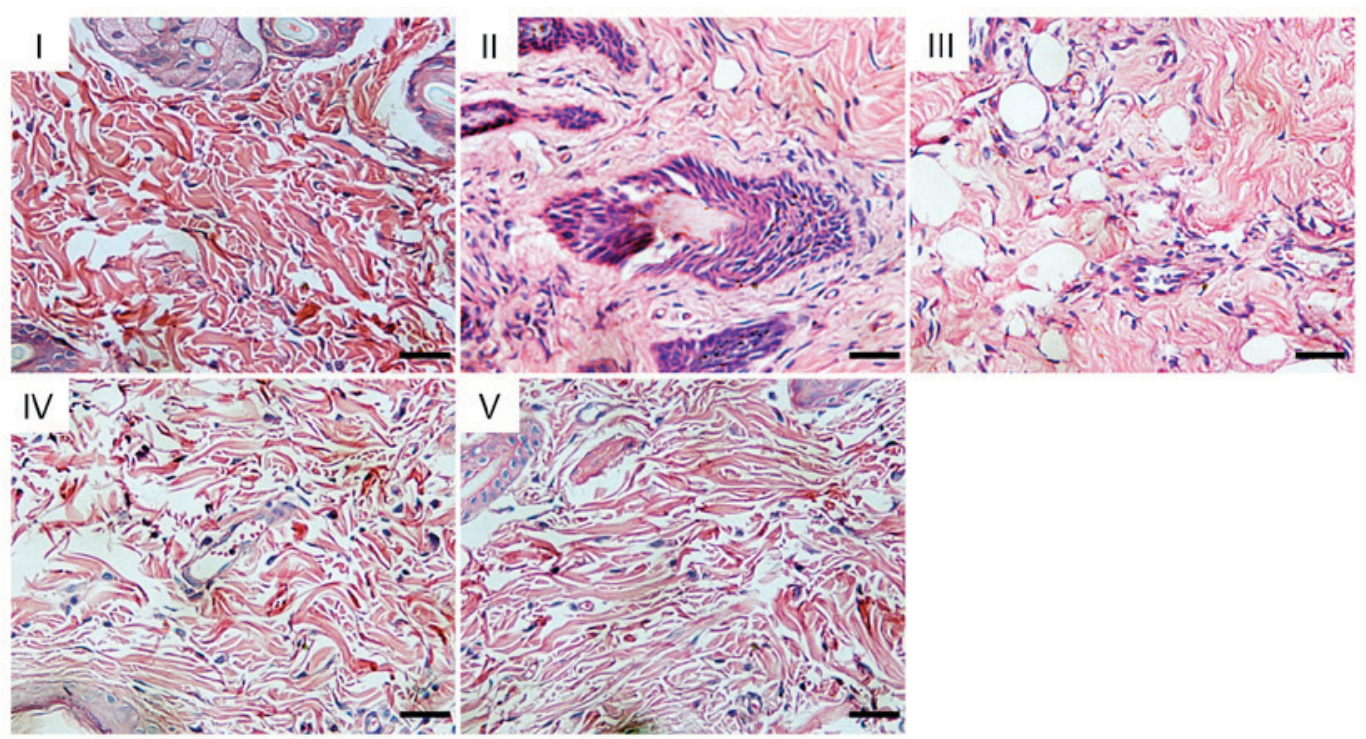

Figure 2. Hematoxylin and eosin-stained sections of skin flap tissue at 7 days after I/R surgery. Magnification, $\mathrm{x} 400$. Scale bar, $50 \mu \mathrm{m}$. The tissue was filled with a large number of neutrophils in group II and III, with only a small amount in group I, IV and V. Control group (group I), I/R group (group II), saline group (group III), estradiol group (group IV), inhibitor group (group V). I/R, ischemia/reperfusion.

Correlation analysis. The Pearson coefficient of correlation analysis ( $\mathrm{r}=-0.595, \mathrm{P}=0.01)$ showed a significant negative correlation between apoptosis index and survival rate of skin flap (Fig. 5).

\section{Discussion}

The current study demonstrated that estradiol therapy reduced the I/R injury of an axial flap. Additionally, inhibition of
ERK signaling partially abolished the effect of estradiol, which suggested that the protective effect of estradiol may be mediated through the ERK pathway. The findings also demonstrated that apoptosis has an important role in I/R injury and inhibition of apoptosis may be an important strategy for the treatment of I/R injury.

$\mathrm{I} / \mathrm{R}$ injury is the dysfunction and structural damage to tissues or organs caused flowing the loss and recovery of blood flow (19). A previous report indicated that the necrosis 


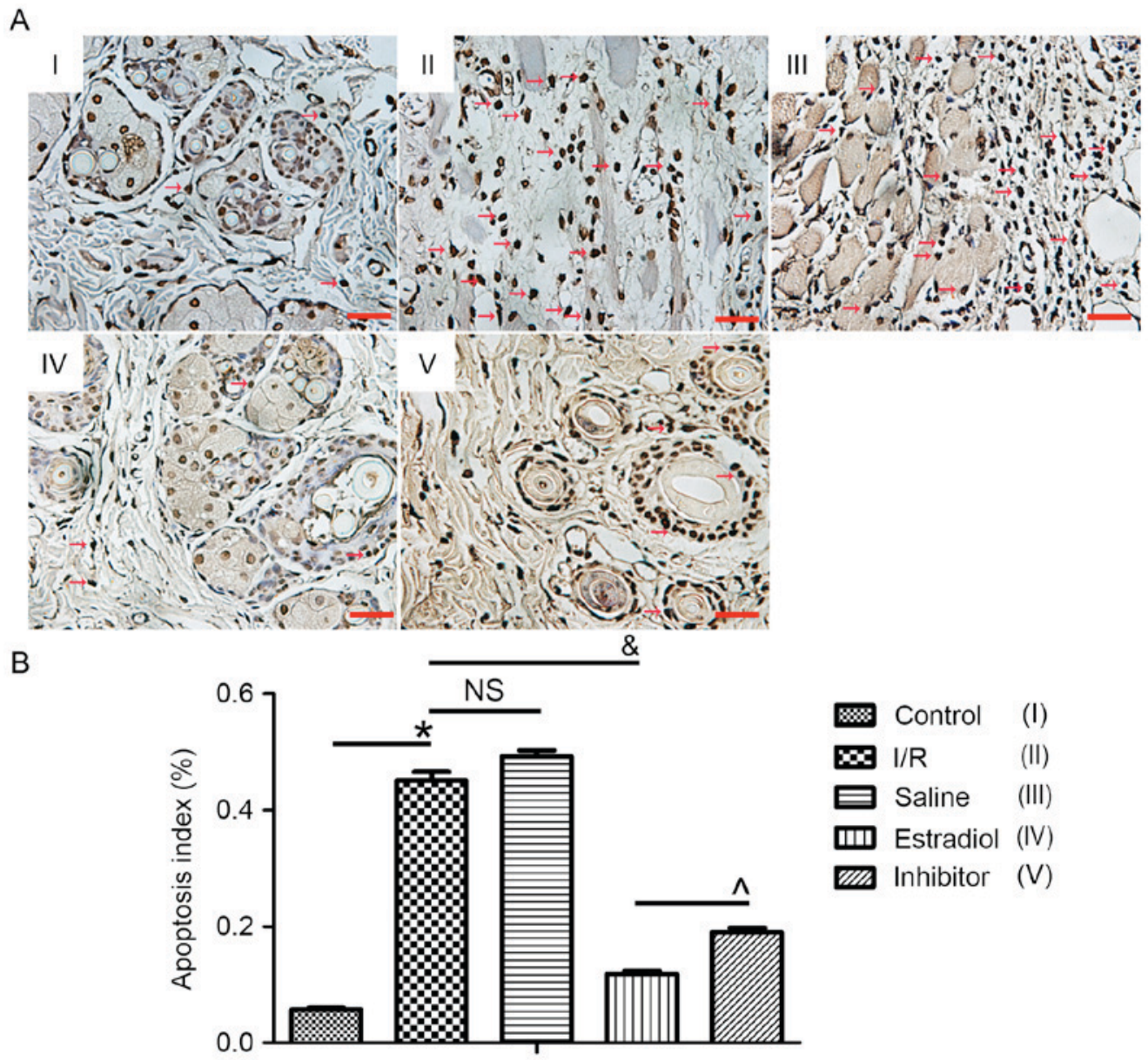

Figure 3. (A) Skin flap samples were obtained 7 days after surgery and terminal deoxynucleotidyl transferase dUTP nick-end labeling was performed. Small red arrows indicated the positive apoptotic cells (magnification, $\mathrm{x} 400$; Scale bar, $50 \mu \mathrm{m}$ ). (B) The apoptosis index of all groups. Three random high-power fields were imaged from each section. Values were expressed as the mean \pm standard deviation. ( $\mathrm{n}=30$, each group had 10 rats, 3 sections per rat; ${ }^{*} \mathrm{P}<0.05$ vs. control; ${ }^{\&} \mathrm{P}<0.05$ vs. I/R; ${ }^{\wedge} \mathrm{P}<0.05$ vs. estradiol. I/R, ischemia/reperfusion; NS, no significance).

rate in pedicle flaps following $\mathrm{I} / \mathrm{R}$ injury was $5-10 \%$ (20). Lipid peroxides, reactive oxygen species, toxic substances and severe inflammatory reaction in patients produced by I/R injury may prolong the duration of patient hospitalization, increase the number of operations require and increase treatment costs, which directly influences the treatment effects of surgery and increases the medical burden of patients (1). However, not all of the ischemic tissue or organs may be damaged after the recovery of blood flow. Various factors affect the occurrence and development of I/R injury, including ischemia duration, collateral circulation, oxygen demand and reperfusion conditions (21). The process of skin flap I/R injury involves a variety of mechanisms to cooperate with each other, which forms a series of linked cascade reaction, resulting in the increased I/R injury and the tissue damage of the skin flap. If the chain reaction is not terminated in a timely manner, the survival rate of the flap is greatly threatened. Therefore, how to use multifactor interventions or drug preconditioning methods to terminate or inhibit certain pathways involved in $\mathrm{I} / \mathrm{R}$ injury is currently a focus of basic and clinical research in this area.

Estrogen, a female hormone, not only dominates the development and maintenance of female reproductive organs and secondary sex characteristic, but also promotes the retention of water and sodium, the deposition of calcium in bones, and influences the endocrine system, cardiovascular system, metabolism and bone growth. Previous research has demonstrated that estrogen reduces I/R injury in the brain, heart and liver (22-24). Furthermore, estrogen may to improve the level of NO and increase the survival area of random skin flaps (25). In the current study, inflammation was reduced, apoptosis was inhibited and the survival area of the axial flap was increased in the rats that were treated with estradiol. The results suggested that estradiol had the similar effect in the axial flap as in previous studies. Thus, similar experiments should be performed applying estrogen to free flaps.

Apoptosis is an independent, orderly and natural death process. The occurrence of apoptosis in tissues following $\mathrm{I} / \mathrm{R}$ is associated with the severity of ischemia and reperfusion time. Numerous reports have demonstrated that apoptosis is mediated by multiple mechanisms during I/R injury (26-28). ROS generated in the I/R-injured area may damage the DNA and mitochondria, and induce the lipid peroxidation of the cell membrane, which affects signal transduction. Additionally, protein cross-linking causes reduce enzyme activities and altered gene transcription, thus resulting in cell apoptosis. Furthermore, inactivation of $\mathrm{Bcl}-2$ protein is induced by calcium overload and a variety of $\mathrm{Ca}^{2+}$-dependent endonucleases that 
A

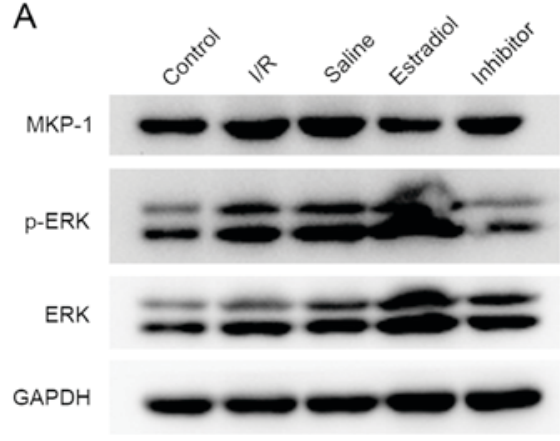

C

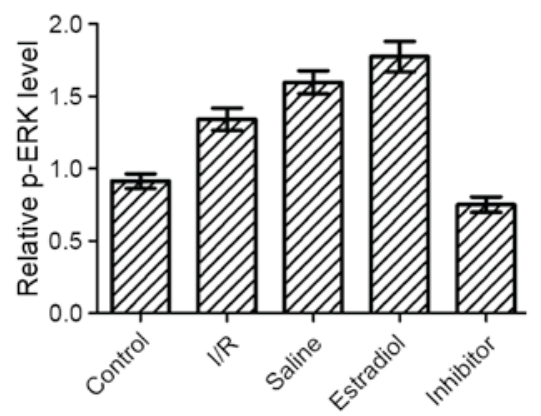

B

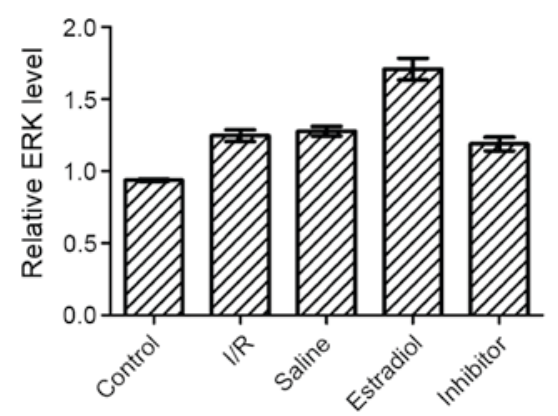

D

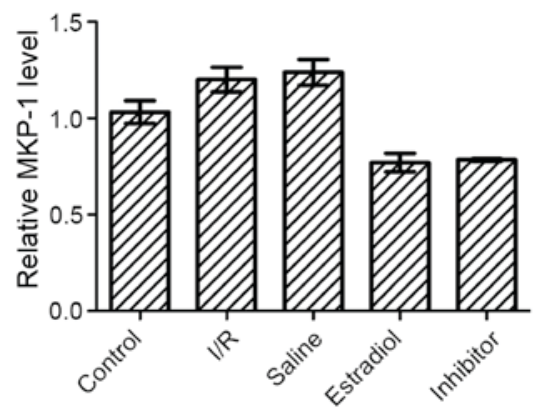

Figure 4. (A) Representative western blot of tissue samples taken from rat skin flaps 7 days after I/R. Relative level of (B) ERK, (C) p-ERK relative to total ERK/GAPDH and (D) MKP-1 protein. I/R, ischemia/reperfusion; ERK, extracellular signal-regulated kinase; p-, phosphorylated; MKP-1, mitogen-activated protein kinase phosphatase 1. Western blot detection was performed in each group, and six in ten groups had the trend of above picture. Each experiment was repeated at least 3 times.

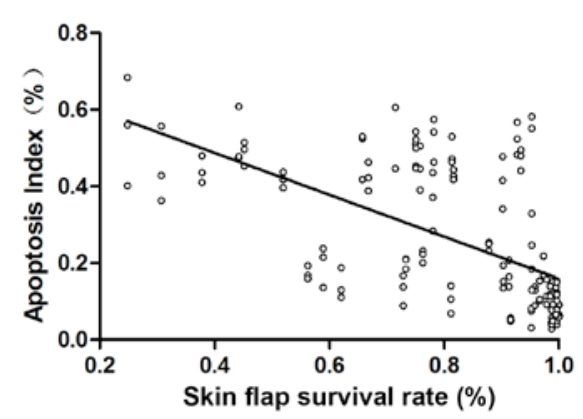

Figure 5. Pearson coefficient of correlation analysis between apoptosis index and survival rate of skin flap.

induce the degradation of DNA, activate tumor necrosis factor signal transduction pathway, mediate the p53 pathway and promote the apoptosis (29). An association between apoptosis and the survival rate of the axial flap was observed in the current study. Almost no hair growth was observed on the flap surface in groups that had high levels of apoptosis after $I / R$ injury, which was detected by TUNEL. Skin ulceration and necrosis were observed at the flap edge following I/R injury. However, less apoptosis was detected in the flaps of rats that had receive estradiol injection, and the hair growth on its surface was close to normal, suggesting that estradiol reduces the apoptosis induced by $\mathrm{I} / \mathrm{R}$ injury in skin flaps.

With the development of molecular biology, more research on estradiol has provided a deeper understanding of the signaling pathway. It was previously reported that estrogen stimulation causes rapid and transient activation of the MAPK protein kinase, that occurred within a few min (30). Another study reported that the estradiol receptor is closely associated with the activity of p38 MAPK, and estradiol can regulate the inflammatory response by regulating the activity of the p38 pathway (31). In brain astrocytes, osteoblasts and glioma cells, ERK signaling is rapidly activated by estradiol $(32,33)$. By complaining between the control group and the other I/R groups, the present demonstrated that the ERK pathway was activated in this experiment. The level of ERK also was increased by estradiol, which had a direct impact on the survival rate of skin flap. In addition, when the ERK pathway was inhibited by PD98059, the survival rate of skin flap was partially reduced and the apoptosis rate was increased. It demonstrated that ERK pathway has an important role in the effect of estradiol.

It has also been reported that an imbalance in expression between MKP-1 and ERK may be one of the important mechanisms that mediate the occurrence and development of tumors (34). Serini et al (35) reported that the mechanism of apoptosis in lung cancer cells induced by docosahexaenoic acid was associated with the increased expression of MKP-1 and downregulation of the ERK pathway. The expression of MKP-1 in the I/R group and saline group were increased in the current model, which may be caused by the stress of I/R injury. Whereas the expression of MKP-1 in the estradiol group was reduced compared with the other three groups, and the levels of ERK and p-ERK were increased, which indicates that the effect of estrogen on ERK pathway may be mediated through MKP-1.

In summary, the findings of the current study suggest that estradiol reduces I/R injury in an axial skin flap. It was 
also demonstrated that the ERK pathway and apoptosis have important roles in the effect of estradiol in this model. However, inhibition of the ERK pathway combined with estradiol treatment only partially reduced the effect of estradiol on the survival rate of the flap, indicating that there are other factors and pathways involved in the effect of estradiol on the survival the skin flap in this model. Further studies are required to determine the precise mechanisms, and to provide novel methods for clinical treatment of I/R injury.

\section{Acknowledgements}

We would like to thank Mrs Jing Wang (Laboratory Animal Center, Soochow University, Suzhou, China) for supplying the experimental animals, and Dr Shiliang Wu (Molecular and Biochemical Institute, Soochow University) for his technical guidance.

\section{References}

1. Rand-Luby L, Pommier RF, Williams ST, Woltering EA, Small KA and Fletcher WS: Improved outcome of surgical flaps treated with topical dimethylsulfoxide. Ann Surg 224: 583-590, 1996.

2. Ozmen S, Ayhan S, Demir Y, Siemionow M and Atabay K: Impact of gradual blood flow increase on ischaemia-reperfusion injury in the rat cremaster microcirculation model. J Plast Reconstr Aesthet Surg 61: 939-948, 2008.

3. Cetin C, Kse AA, Aral E, Colak O, Erçel C, Karabağli Y, Alataş $\mathrm{O}$ and Eker A: Protective effect of fucoidin (a neutrophil rolling inhibitor) on ischemia reperfusion injury: Experimental study in rat epigastric island flaps. Ann Plast Surg 47: 540-546, 2001 .

4. Molski M, Groth A, Allison AC, Hendrickson M and Siemionow M: Diannexin treatment decreases ischemia-reperfusion injury at the endothelial cell level of the microvascular bed in muscle flaps. Ann Plast Surg 63: 564-571, 2009.

5. Hu X, Xu W and Jiang H: HMGB1/IL-17A axis: An important mechanism for myocardial ischemia-reperfusion injury. Int J Cardiol 174: 447-448, 2014

6. Shen B, Zhou S, He Y, Zhao H, Mei M and Wu X: Revealing the underlying mechanism of ischemia reperfusion injury using bioinformatics approach. Kidney Blood Press Res 38: 99-108, 2013.

7. Burns AT, Davies DR, McLaren AJ, Cerundolo L, Morris PJ and Fuggle SV: Apoptosis in ischemia/reperfusion injury of human renal allografts. Transplantation 66: 872-876, 1998.

8. Rossing L, Haendeler J, Mallat Z, Hugel B, Freyssinet JM, Tedgui A, Dimmeler S and Zeiher AM: Congestive heart failure induces endothelial cell apoptosis: Protective role of carvedilol Journal. Am Coll Cardiol 36: 2081-2089, 2003.

9. Messmer UK, Briner VA and Pfeilschifter J: Basic fibroblast growth factor selectively enhance TNF-alpha induced apoptotic cell death in glomerular endothelial cells: Effects on apoptotic signaling pathways. J Am Soc Nephrol 11: 2199-2211, 2000.

10. Chong H, Vikis HG and Guan KL: Mechanisms of regulating the Raf kinase family. Cell Signal 15: 463-469, 2003.

11. Mercer KE and Pritchard CA: Raf proteins and cancer: B-Raf is identified as a mutational target. Biochim Biophys Acta 1653: 25-40, 2003.

12. Abe J, Baines CP and Berk BC: Role of mitogen-activated protein kinases in ischemia and reperfusion injury: The good and the bad. Circ Res 86: 607-609, 2000.

13. Darling CE, Jiang R, Maynard M, Whittaker P, Vinten-Johansen J and Przyklenk K: Postconditioning via stuttering reperfusion limits myocardial infarct size in rabbit hearts: Role of ERK1/2. Am J Physiol Heart Circ Physiol 289: 1618-1626, 2005.

14. Jung JY, Yoo CI, Kim HT, Kwon CH, Park JY and Kim YK: Role of mitogen-activated protein kinase (MAPK) in troglitazone-induced osteoblastic cell death. Toxicology 234: 73-82, 2007.

15. Nunes-Xavier C, Romá-Mateo C, Ríos P, Tárrega C, Cejudo-Marín R, Tabernero L and Pulido R: Dual-specificity MAP kinase phosphatases as targets of cancer treatment. Anticancer Agents Med Chem 11: 109-132, 2011.
16. Diederich RS, Mowlavi A, Meldrum G, Medling B, Bueno RA and Neumeister MW: Local cooling provides muscle flaps protection from ischemia-reperfusion injury in the event of venous occlusion during the early reperfusion period. Hand (N Y) 4: 19-23, 2009.

17. Chen Z, Yuhanna IS, Galcheva-Gargova Z, Karas RH, Mendelsohn ME and Shaul PW: Estrogen receptor alpha mediates the nongenomic activation of endothelial nitric oxide synthase by estrogen. J Clin Invest 102: 401-406, 1999.

18. Manson GF, Anthenelli RM, Im MJ, Bulkley GB and Hoopes JE: The role of oxygen-free radicals in ischemia tissue injury in island skin flaps. Ann Surg 198: 87-90, 1983.

19. Zingarelli B: Ischemia-reperfusion injury. In: Wheeler DS, Wong HR, Shanley TP (eds). Science and Practice of Pediatric Critical Care Medicine. London, Springer-Verlag, pp181-192, 2009.

20. Harder Y, Amon M, Laschke MW, Schramm R, Rücker M, Wettstein R, Bastiaanse J, Frick A, Machens HG, Küntscher M, et al: An old dream revitalised: Preconditioning strategies to protect surgical flaps from critical ischaemia and ischaemia-reperfusion injury. J Plast Reconstr Aesthet Surg 61: 503-511, 2008.

21. Montalvo-Jave EE, Escalante-Tattersfield T, Ortega-Salgado JA, Piña E and Geller DA: Factors in the pathophysiology of the liver ischemia-reperfusion injury. J Surg Res 147: 153-159, 2008

22. Xu HL, Baughman VL and Pelligrino DA: Estrogen replacement treatment in diabetic ovariectomized female rats potentiates poetischemic leukocyte adhesion in cerebral venules. Stroke 35: 1974-1978, 2004.

23. Carswell HV, Dominiczak AF and Macrae IM: Estrogen status affects sensitivity to focal cerebral ischemia in stroke-prone spontaneously hypertensive rats. Am J Physiol Heart Cire Physiol 278: 290-294, 2000.

24. Shen SQ, Zhang Y and Xiong CL: The protective effects of 17beta-estradiol on hepatic ischemia-reperfusion injury in rat model, associated with regulation of heat-shock protein expression. J Surg Res 140: 67-76, 2007.

25. Shafighi M, Fathi AR, Brun C, Huemer GM, Wirth R, Hunger R, Banic A and Constantinescu MA: Topical application of $17 \beta$-estradiol (E2) improves skin flap survival through activation of endothelial nitric oxide synthase in rats. Wound Repair Regen 20: 740-747, 2012.

26. Jia D, Han B, Yang S and Zhao J: Anemonin alleviates nerve injury after cerebral ischemia and reperfusion $(\mathrm{i} / \mathrm{r})$ in rats by improving antioxidant activities and inhibiting apoptosis pathway. J Mol Neurosci 53: 271-279, 2014.

27. Koshinuma S, Miyamae M, Kaneda K, Kotani J and Figueredo VM: Combination of necroptosis and apoptosis inhibition enhances cardioprotection against myocardial ischemia-reperfusion injury. J Anesth 28: 235-241, 2014.

28. Tao T, Chen F, Bo L, Xie Q, Yi W, Zou Y, Hu B, Li J and Deng X: Ginsenoside Rg1 protects mouse liver against ischemia-reperfusion injury through anti-inflammatory and anti-apoptosis properties. J Surg Res 191: 231-238, 2014.

29. Scarabelli TM and Gottlieb RA: Functional and clinical repercussions of myocyte apoptosis in the multifaceted damage by ischemia/reperfusion injury: Old and new concepts after 10 years of contributions. Cell Death Differ 11 (Suppl 2): S144-S152, 2004.

30. Collins P and Webb C: Estrogen hits the surface. Nat Med 5: 1130-1131, 1999.

31. Bhatt S, Xiao Z, Meng Z and Katzenellenbogen BS: Phosphorylation by p38 mitogen-activated protein kinase promotes estrogen receptor a tumor and functional activity via the SCF (Skp2) proteasomal complex. Mol Cell Biol 32: 1928-1943, 2012.

32. Ivanova TV, Ivanov VN and Nadezhdina ES: Transcription factors NFkappaB and AP-1/c-fos incell response to nocodazol. Membr Cell Biol 14: 727-741, 2001.

33. Endoh H, Sasaki H, Maruyama K, Takeyama K, Waga I, Shimizu T, Kato S and Kawashima H: Rapid activation of MAP kinase by estrogen in the bone cell line. Biochem Biophys Res Commun 235: 99-102, 1997.

34. Guo X, Zhang X, Li Y, Guo Y, Wang J, Li Y, Shen B, Sun D and Zhang J: Nocodazole increases the ERK activity to enhance MKP-1 expression which inhibits p38 activation induced by TNF- $\alpha$. Mol Cell Biochem 364: 373-380, 2012.

35. Serini S, Trombino S, Oliva F, Piccioni E, Monego G, Resci F, Boninsegna A, Picci N, Ranelletti FO and Calviello G: Docosahexaenoic acid induces apoptosis in lung cancer cells by increasing MKP-1 and down-regulating p-ERK1/2 and p-p38 expression. Apoptosis 13: 1172-1183, 2008. 